\title{
Immunoprophylaxis and therapy of grafted rat colonic carcinoma
}

\author{
M. S. MARTIN1, F. MARTIN, E. JUSTRABO, M. F. MICHEL, AND A. LAGNEAU \\ From the Laboratory of Immunology, the Laboratory of Pathology and INSERM, Faculté de Médecine, \\ Dijon, France
}

SUMMARY Two independent lines of chemically-induced colonic carcinoma, serially graftable in syngeneic rats, have been used to investigate the effects of immunoprophylaxis and immunotherapy. Rats were immunised by various procedures, including BCG, irradiated tumour grafts, and cancer cells treated by mitomycin and neuraminidase. A partial inhibition of tumour growth was observed in one of the four tested protocols. On the other hand, a significant enhancement of tumour growth was obtained in two other experiments.

Patients with colon carcinoma have been shown to react immunologically against tumour-associated antigens (Hellström et al., 1970; Hollinshead et al., 1970; Baldwin et al., 1973). This finding suggests the use of immunotherapy to secure the surgical cure of this tumour. Demonstration of the objective effects of immunotherapy and the choice of the most efficient method require prolonged study in humans, because of the slow evolution of colorectal cancer. For this reason, we have chosen an animal model of chemically-induced colonic cancer (Martin et al., 1973a). The following report deals with our preliminary attempts to treat rat colonic cancer by specific or non-specific immunological treatment.

\section{Methods}

\section{ANIMALS}

All rats were of the inbred BD-IX strain, maintained by continuous brother-sister mating. Inbreeding has been confirmed by permanent acceptance of skin grafts.

\section{TUMOURS}

Two serially graftable tumour lines, DHB and DHD, were independently obtained from colonic adenocarcinomas induced by 1,2 dimethylhydrazine (Martin et al., 1973b). Cell lines have been established by trypsinisation of the grafted tumours and

\footnotetext{
${ }^{1}$ Address for reprints: M. S. Martin, Laboratoire d'Immunologie, Faculté de Médecine, 21000, Dijon, France.

Received for publication 20 September 1976
}

growth as pure epithelial-like colonies, serially passaged after $0.25 \%$ trypsin dissociation (Martin et al., 1975).

\section{CHALLENGING CELLS}

They were obtained either by $0.25 \%$ trypsinisation of established cell cultures (experiments 1,2, and 3), or by dissociation of freshly removed tumour grafts, using $0.25 \%$ pronase and $0.005 \%$ desoxyribonuclease, according to Bartlett and Zbar (1972) (experiment 4). In both cases only living cells, excluding blue Trypan (which were usually more than $90 \%$ ), were taken into account. Challenging cells were subcutaneously injected into the anterior thoracic wall at the concentration of $2 \times 10^{6}$ cells $/ \mathrm{ml}$.

\section{IRRADIATION}

Tumour grafts (about $5 \times 5 \times 5 \mathrm{~mm}$ ) were irradiated $(15,000$ rads) for about 15 minutes in an electron beam of $7 \mathrm{MeV}$ produced by a linear accelerator (Sagitaire, CSF, France).

TREATMENT OF CELLS BY MITOMYCIN AND NEURAMINIDASE

DHB or DHD cells, obtained by trypsinisation of cell cultures, were incubated for one hour at $37^{\circ} \mathrm{C}$ in Ham's F 10 medium (Gibco, Grand Island, USA) containing Vibrio cholerae neuraminidase (Behringwerke, Marburg-Lahn, Germany) at a concentration of $25 \mathrm{U} / \mathrm{ml} / 10^{6}$ cells and mitomycin C (Sigma Chemical Company, St. Louis, USA) at a concentration of $25 \mu \mathrm{g} / \mathrm{ml} / 10^{6}$ cells. Cells were washed twice in phosphate buffered saline before being injected into the animals. 
BCG

Mycobacterium bovis, strain BCG, was obtained lyophilised from the Institut Pasteur, Paris, France. Just before the injection, it was diluted to a concentration of $5 \mathrm{mg} / \mathrm{ml}$ (about $30 \times 10^{6}$ viable units per $\mathrm{ml}$ ).

\section{STATISTICAL ANALYSIS}

Treated and control groups were compared using the Fischer exact probability test for tumour incidence and the Mann and Whitney $U$ test for tumour weight.

\section{Results}

\section{EXPERIMENT 1}

A group of 21 rats received monthly irradiated DHB grafts for six months, then two subcutaneous injections of $10^{6}$ mitomycin- and neuraminidasetreated DHB cells. A paired group of control animals was simultaneously sham-grafted, then injected with Ham's F 10 medium. Three weeks after the last injection, surviving animals (18 immunised and 17 controls) were challenged with subcutaneous injection of $10^{6}$ viable DHB cells. All the animals were killed 35 days later. Tumours developed in 10 out of the 17 controls and in all the 18 immunised rats $(P=0,006)$. Mean tumour weight was $0.4 \mathrm{~g}$ in controls and $3.1 \mathrm{~g}$ in treated animals $(\mathrm{P}<0.01)$.

\section{EXPERIMENT 2}

Another group of eight rats received three intraperitoneal injections at two week intervals of $2 \times 10^{6}, 4 \times 10^{6}$, and $2 \times 10^{6}$ DHD cells treated by mitomycin and neuraminidase. Two weeks after the last injection, this group and paired controls were injected subcutaneously with $10^{6}$ untreated DHD cells. They were killed 50 days later. The tumour incidence was the same (five out of eight) in both groups. The mean tumour weight was $244 \mathrm{mg}$ in the control group and $820 \mathrm{mg}$ in the treated group (not significant).

\section{EXPERIMENT 3}

Two groups of 10 rats were challenged by subcutaneous injection of $10^{6}$ viable DHD cells. Ten days later, one group received a series of six subcutaneous injections (every other day) of $2.5 \mathrm{mg}$ BCG mixed with $10^{6}$ mitomycin- and neuraminidasetreated DHD cells. Animals of both groups were killed 122 days after the challenge. Tumours were found in seven out of 10 controls and all the 10 treated animals (not significant). The mean tumour weight was $1.6 \mathrm{~g}$ in controls and $10.9 \mathrm{~g}$ in immunised rats $(P<0.01)$. Lung metastases occurred in one control and eight treated rats $(P=0.005)$.

\section{EXPERIMENT 4}

Seven groups of eight rats were challenged on the same day by subcutaneous injection of $10^{6}$ viable DHD cells obtained by enzymatic dissociation of freshly removed tumour grafts. One group received no other treatment (control group). The six other groups were inoculated with $9.5 \mathrm{mg} \mathrm{BCG}$ in $0.5 \mathrm{ml}$ phosphate buffered saline at different times before or after challenge. BCG was always injected at the exact challenge site or into the tumour when a cancer nodule was detectable. The local inflammatory reaction induced by BCG was usually mild, limited to a small subcutaneous nodule, disappearing 15 to 25 days after BCG inoculation. Group I received $\mathrm{BCG}$ seven days before challenge. In group II, BCG was inoculated together with the tumour cells. In group III, IV, V, and VI, BCG was given respectively five, 10,15 , and 20 days after challenge. Tumour grew in all control animals with palpable growth being observed between 10 and 15 days after inoculation. All the rats were killed on the 56th day after the challenge. Results of the experiment are summarised in the Table. The number of

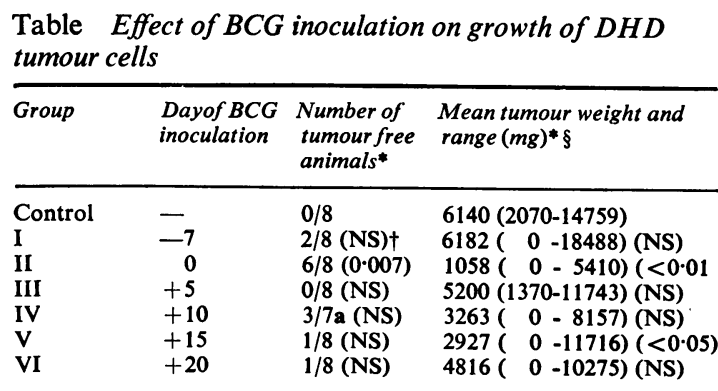

*Significance when compared with the control group.

†NS: not significant.

$\ddagger$ One rat died from intercurrent infection four days after BCG inoculation.

\$The mean tumour weights include data from animals in which no tumours grew.

tumour-free animals was not significantly different from the control group except for group II (BCG given together with the tumour cells), in which six out of eight rats were tumour-free at the end of the experiment. The mean tumour weight was smaller in the rats receiving BCG after tumour cells injection; the difference was significant for group V (BCG inoculated 15 days after challenge).

\section{Discussion}

In the four reported experiments, the effect of 
immunological treatment on the growth of grafted colonic tumours was rather disappointing. The one favourable effect was obtained in experiment 4, where BCG alone was used as immunostimulating agent. In this experiment, tumour growth was slower when BCG was injected into an established tumour and was often suppressed when BCG was inoculated together with the challenging cells. Suppression of tumour growth by the injection of tumour cells admixed with BCG has previously been reported by Baldwin and Pim (1973) and Kreider et al. (1976); unfortunately, this suppressive effect has no clinical equivalence.

On the other hand, significant enhancement of tumour growth was demonstrated in experiments 1 and 3 , where tumour cells, or tumour cells plus BCG, were used for immunostimulatory therapy. This enhancement was surprisingly great as the tumours were approximately seven times larger in treated animals than in controls. The increased incidence of lung metastases observed in experiment 3 was also highly significant.

The factor responsible for tumour enhancement could not be defined. Serial immunisation with irradiated $(15,000 \mathrm{r})$ tumour grafts, which gives enhancement in our experiment 1 , was successfully used by Baldwin and Barker (1967) to prevent growth of rat hepatoma. Simmons and Rios (1973) were able to cure 24 out of 42 fibrosarcomas established in mice by repeated injections of BCG plus mitomycine- and neuraminidase-treated tumour cells; their protocol was very similar to that used in our experiment 3. Enhancement of tumour growth by BCG and/or neuraminidase treated cells has previously been reported (Piessens et al., 1970; Baldwin and Pim, 1973; Sparks and Breeding, 1974), although the conditions leading to protection or enhancement have never been clearly determined. It has been suggested that the effectiveness of BCGinduced suppression of tumours depends on the immunogenicity of the target tumour (Baldwin and Pim, 1973). Cancers with less immunogenic capacity could be enhanced rather than suppressed. Although the transplantation immunity of DHB and DHD tumour lines has not yet been systematically tested, these tumours are definitely able to induce an immune response in the tumour-bearing host. Mcmbrane immunofluorescence technique detects antibodies in a large proportion of animals grafted with these tumours (Martin et al., 1976). Steele and Sjögren (1974) demonstrated that various rat colonic carcinomas shared a surface antigen inducing lymphocyte-mediated cytotoxicity. However, the effect of this antigen in tumour prevention could be inhibited by circulating blocking factors (Steele $e t$ al., 1975).
It is also possible that rat colonic carcinoma is particularly susceptible to immunological enhancement. This hypothesis agrees with data from Rogers and Gildin (1975) on the effects of BCG treatment on dimethylhydrazine-induced rat colon cancer: BCG injected into the intestinal wall did not significantly alter the incidence of colonic carcinomas, but increased the percentage of animals with metastases.

Even if results obtained in animal models must be extrapolated with caution, present data emphasise the potential hazards of tumour enhancement in the immunotherapy of human colorectal cancer. At the present time, a few trials of immunotherapy of human intestinal cancer have been reported (Moertel et al., 1975; Falk et al., 1976; Mavligit et al., 1976). They were not conclusive, mainly because the patients were not randomised before being entered in the trial. Additional experimental studies, using different immunisation procedures, could help the clinician to define the factors leading to suppression, instead of enhancement, of human colorectal cancer growth.

We wish to thank Dr J. C. Horiot and Dr R. Chomono for irradiation of tumour grafts and $\mathrm{Dr} S$. Jampolis for revision of the manuscript. This work was supported by research grants 74.1.080.7 of Institut National de la Santé et Recherche Médicale and 74.70495 of Délégation Générale à la Recherche Scientifique et Technique.

\section{References}

Baldwin, R. W., and Barker, C. R. (1967). Tumour specific antigenicity of aminoazo dye-induced rat hepatomas. International Journal of Cancer, 2, 355-364.

Baldwin, R. W., Embleton, M. J., Jones, J. S. P., and Langman, M. J. S. (1973). Cell-mediated and humoral immune reactions to human tumours. International Journal of Cancer, 12, 73-83.

Baldwin, R. W., and Pimm, M. V. (1973). BCG immunotherapy of rat tumors of defined immunogenicity. National Cancer Institute Monographs, 39, 11-17.

Bartlett, G. L., and Zbar, B. (1972). Tumor-specific vaccine containing Mycobacterium bovis and tumor cells: safety and efficacy. Journal of the National Cancer Institute, 48, 1709-1726.

Falk, R. E., MacGregor, A. B., Landi, S., Ambus, U., and Langer, B. (1976). Immunostimulation with intraperitoneally administered Bacille Calmette-Guérin for advanced malignant tumors of the gastrointestinal tract. Surgery, Gynecology and Obstetrics, 142, 363-368.

Hellström, I., Hellström, K. E., and Shepard, T. (1970). Cell-mediated immunity against antigens common to human colonic carcinomas and fetal gut epithelium. International Journal of Cancer, 6, 346-351.

Hollinshead, A., Glew, D., Bunnag, B., Gold, P., and Herberman, R. (1970). Skin-reactive soluble antigen from intestinal cancer-cell membranes and relationship to carcinoembryonic antigens. Lancet, 1, 1191-1195.

Kreider, J. W., Bartlett, G. L., and Purnell, D. M. (1976). Suitability of rat mammary adenocarcinoma 13762 as a model for BCG immunotherapy. Jourral of the National Cancer Institute, 56, 797-802. 
Martin, M. S., Bastien, H., Martin, F., Michiels, R., Martin, M. R., and Justrabo, E. (1973b). Transplantation of intestinal carcinoma in inbred rats. Biomedicine, 19, 555-558.

Martin, F., Knobel, S., Martin, M., and Bordes, M. (1975). A carcinofetal antigen located on the membrane of cells from rat intestinal carcinoma in culture. Cancer Research, 35, 333-336.

Martin, F., Martin, M., Lagneau, A., Bordes, M., and Knobel, S. (1976). Circulating antibodies in rats bearing grafted colon carcinoma. Cancer Research 36, 30393042.

Martin, M. S., Martin, F., Michiels, R., Bastien, H., Justrabo, E., Bordes, M., and Viry, B. (1973a). An experimental model for cancer of the colon and rectum: intestinal carcinoma induced in the rat by 1, 2-dimethylhydrazine. Digestion, 8, 22.34.

Mavligit, G. M., Gutterman, J. U., Burgess, M. A., Khankhanian, N., Seibert, G. B., Speer, J. F., Jubert, A. V., Martin, R. C., McBride, C. M., Copeland, E. M., Gehan, E. A., and Hersh, E. M. (1976). Prolongation of postoperative disease-free interval and survival in human colorectal cancer by BCG or BCG plus 5-fluorouracil. Lancet, 1, 871-876.

Moertel, C. G., Ritts, R. E., Schutt, A. J., and Hahn, R. G. (1975). Clinical studies of methanol extraction residue fraction of Bacillus Calmette-Guérin as an immunostimulant in patients with advanced cancer. Cancer Research, 35, 3075-3083.

Piessens, W. F., Lachapelle, F. L., Legros, N., and Heuson, J. (1970). Facilitation of rat mammary tumor growth by BCG. Nature, 228, 1210-1211.

Rogers, A. E., and Gildin, J. (1975). Effect of BCG on dimethylhydrazine induction of colon tumors in rats. Journal of the National Cancer Institute, 55, 385-391.

Simmons, R. L., and Rios, A. (1973). Comparative and combined effect of BCG and neuraminidase in experimental immunotherapy. National Cancer Institute Monographs, 39, 57-65.

Sparks, F. C., and Breeding, J. H. (1974). Tumor regression and enhancement resulting from immunotherapy with Bacillus Calmette-Guérin and neuraminidase. Cancer Research, 34, 3262-3269.

Steele, G., Jr, and Sjögren, H. O. (1974). Cross-reacting tumor-associated antigen(s) among chemically induced rat colon carcinomas. Cancer Research, 34, 1801-1807.

Steele, G., Jr, Sjögren, H. O., Rosengren, J. E., Lindström, C., Larsson, A., and Leandoer, L. (1975). Sequential studies of serum blocking activity in rats bearing chemically induced primary bowel tumors. Journal of the National Cancer Institute, 54, 959-967. 